\title{
POLYNOMIALLY CONVEX SETS
}

\author{
BY GABRIEL STOLZENBERG
}

Communicated by A. M. Gleason, February 16, 1962

0 . Introduction. This is a report on the polynomial convex hull of a compact set, $X$, in complex $n$-space, $C^{n}$. By definition, it is hull $(X)$, the set of all $p$ in $C^{n}$ such that

$$
|f(p)| \leqq \max _{x \in X}|f(x)|,
$$

for every polynomial, $f\left(z_{1}, \cdots, z_{n}\right)$. When $X=\operatorname{hull}(X)$, we say $X$ is polynomially convex. In studying the polynomial convex hull, it helps to introduce also $R$-hull $(X)$, the rational convex hull of $X$. By definition, $R$-hull $(X)$ is the set of all $p$ in $C^{n}$ such that

$$
|g(p)| \leqq \max _{x \in X}|g(x)|,
$$

for all rational functions, $g$, which are analytic about $X$. Equivalently, $R$-hull $(X)$ may be described as the set of all $p$ in $C^{n}$ for which $f(p)$ belongs to $f(X)$, for every polynomial, $f$. When $X=R$-hull $(X)$, we say that $X$ is rationally convex. Evidently,

$$
X \subset R \text {-hull }(X) \subset \operatorname{hull}(X),
$$

and these hulls are compact.

Polynomially convex sets occur prominently in the theory of uniform approximation. Every finitely-generated function algebra can be realized as the uniform closure of the polynomials on a compact subset, $X$, of some $C^{n}$; in which case, its maximal ideal space is precisely $\operatorname{hull}(X)[13]$.

I. Local descriptions of the hulls. To begin, we explain what we mein by a curve of analytic hypersurfaces in an open subset, $O$, of $C^{n}$. If $U$ is a domain in $O$ and $F_{t}, 0 \leqq t<1$, is a curve of nonconstant analytic functions on $U$, we let $H_{t}$ be the zero-set of $F_{t}$ in $U$. If each $H_{t}$ is closed in $O$, then we say that $\left(F_{t}, H_{t}\right)$ is a curve of analytic hypersurfaces in $O$. We shall denote it, simply, by $\left(H_{t}\right)$.

Almost all the results of $\S \S I$ and II are based on the following beautiful local characterization of the polynomial convex hull, given by $\mathrm{K}$. Oka in 1937 , in [6].

(I.0) Oka's Characterization theOREM. Let $O$ be a neighborhood of hull $(X)$ in $C^{n}$. If $\left(H_{t}\right)$ is a curve of analytic hypersurfaces in $O$ such 
that some $H_{t}$ intersects hull $(X)$, but some other $H_{t^{\prime}}$ does not, then some $H_{t^{\prime \prime}}$ must intersect $X$.

Oka's proof uses both his solution of the Cousin I problem [5], and the Oka-Weil Approximation Theorem [5], as a way of passing, in two steps, from local to global information.

Theorem I.0 yields very easily

(I.1) Rossi's LOCAL MAXIMUM MOdUlus PRINCIPLE. If $Y$ is a subset of hull $(X)-X$, then $Y$ is contained $i n^{1}$ hull $(\partial Y)$ [7]. Moreover, by analyzing the Cousin I problem solved in the proof of Theorem I.0 and appealing to E. Bishop's " $1 / 4-3 / 4$ " description of peak points, ${ }^{2}$ [1], we obtain also

(I.2) Rossi's LOCAL PEAK POINT THEOREM. On hull $(X)$, every local peak point ${ }^{2}$ is a peak point [7].

In the original proofs of Theorems I.1 and I.2, Rossi used a somewhat more difficult argument, based on a solution of a Cousin II problem, ${ }^{3}$ (see [7]), and obtained somewhat stronger results. It happens that, for the corresponding local description of the rational convex hull (Theorem I.3 below), a Cousin II type argument is essential.

(I.3) Theorem. If $Y$ is a subset of $R$-hull $(X)-X$, and if $\check{H}^{2}(R$-hull $(X) ; Z)=0$, then $Y$ is contained in $R$-hull $(\partial Y)$.

The topological restriction is really needed. It can be shown, by an example, that the conclusion of Theorem I.3 may not obtain if $\check{H}^{2}(R$-hull $(X) ; Z) \neq 0$.

II. Applications to simply-coconnected sets. We shall say that $X$ is simply-coconnected when ${ }^{5} \check{H}^{1}(X ; Z)=0$. If $X$ is a compact subset of $C^{n}$, we say that $X$ is polynomially convex in dimension one if ${ }^{6} X \cap V$ is polynomially convex, for every $V$ which is a complex one-dimensional analytic subvariety of $C^{n}$.

(II.0) REMARK. Every simply-coconnected, rationally convex set is polynomially convex in dimension one. However, there do exist simplycoconnected sets which are polynomially convex in dimension one, but which are not rationally convex. (Consider, for example, the nonrationally convex arc described by $\mathrm{J}$. Wermer in [9].)

\footnotetext{
$1 \partial$ denotes topological boundary (in the hull of $X$ ).

2 As defined in $[7$, p. 6], for the uniform closure of the polynomials on hull $(X)$.

${ }^{3}$ However, A. Browder noticed that a Cousin I solution would suffice in I.1.

4 Cech cohomology with integer coefficients.

${ }^{5}$ Equivalently, every map of $X$ into $C^{1}-\{0\}$ has a log.

${ }^{6}$ This is a semi-topological condition, asserting that every component of $V-(X \cap V)$ is unbounded.
} 
All the results of this section will depend on the following

(II.1) Theorem. Let $X$ be a compact set in $C^{n}$ and let $f$ be a polynomial. If a branch of $\log (f)$ is defined on $X$, and $f(X)$ does not intersect $f($ hull $(X)-X)$, then $f$ does not vanish at any point of hull $(X)$.

The proof is based on Oka's Characterization (I.0). Roughly, if $f$ were to vanish at a point of hull $(X)$, then $\log (f)$ would "unwind" $X$ from hull $(X)$. In that case, we study the image, under $\log (f)$, of a neighborhood of $X$, and construct a certain curve of analytic hypersurfaces which would contradict Oka's Characterization.

A direct corollary is

(II.2) THeOREM. If $X$ is a compact, simply-coconnected subset of $C^{n}$, and if there is a polynomial, $f$, such that $f(X)$ does not intersect $f($ hull $(X)-X)$, then $X$ is polynomially convex.

We put our most general application of Theorems II.1 and II.2 in the following way.

(II.3) Theorem. Let $X$ be a compact subset of $C^{n}$ which is simplycoconnected and polynomially convex in dimension one. Suppose there are $n-1$ polynomials, $f_{1}, \cdots, f_{n-1}$, which are in general position on $C^{n}$, and such that, for each $j=1, \cdots, n-1, f_{j}(X)$ is contained in the minimal boundary 8 for the uniform limits of functions analytic about $f_{j}($ hull $(X))$. Then $X$ is polynomially convex.

We next list some very special cases of Theorem II.3. But first, define the $n$-torus, $T(n)=\left\{p \in C^{n}:\left|z_{i}(p)\right|=1, i=1, \cdots, n\right\}$. Then,

(II.4) THEOREM. If $X$ is simply-coconnected and rationally convex (or, if $X$ is an arc) and lies in $T(n-1) \times C^{1}$, then $X$ is polynomially convex.

Since every subset of $T(n)$ is rationally convex, this implies

(II.5) Theorem. If $X$ is contained in a simply-coconnected subset of $T(n)$, then $X$ is polynomially convex. Moreover, every complex-valued continuous function on $X$ is a uniform limit of polynomials.

Also, we have

(II.6) THEOREM. If $X$ is simply-coconnected and rationally convex (or, if $X$ is an arc) and lies in $C^{2}$, and if there is a nonconstant polynomial, $f$, with $|f|=1$ on $X$, then $X$ is polynomially convex.

REMARK. We would be delighted to know the answer to the

7 Their common level sets have complex dimension, at most, one.

${ }^{8}$ Defined in $[1 ; 13]$. In many cases it is identical with $\partial\left(f_{j}(\operatorname{hull}(X))\right)$. 
(II.7) QUEsTION. Is every simply-coconnected, rationally convex set polynomially convex?

Theorem II.2 is as close as we have come to an affirmative answer. (But see also Remark III.3.)

III. Approximation by analytic polyhedra. By an analytic polyhedron, $P$, in $C^{n}$, we shall mean a polynomially convex set of the form

$$
P=\left\{u \in U:\left|f_{j}(u)\right| \leqq k_{j}, j=1, \cdots, r\right\},
$$

where $U$ is an open subset of $C^{n}$, the $f_{j}$ are analytic on $U$, and the $k_{j}$ are non-negative constants.

It is evident, from the very definition of a polynomially convex set, that it can be expressed as a decreasing intersection of analytic polyhedra (where the defining functions are, in fact, polynomials). A far deeper result of E. Bishop, [2, p. 225], shows that, in $C^{n}$, we can arrange that every approximating polyhedron be defined by exactly $n$ inequalities.

By the general theory of function algebras, [13], it is known that, for every compact subset, $X$, of $C^{n}$, there exists $S_{X}$, the unique smallest closed subset for which hull $\left(S_{X}\right)=\operatorname{hull}(X)$. We call $S_{X}$ the Silov boundary of hull $(X)$. For an analytic polyhedron, $P$, in $C^{n}$, defined by precisely $n$ inequalities, $\left|f_{j}\right| \leqq 1, j=1, \cdots, n$, the Silov boundary, $S_{P}$, has an especially pleasing form.

$$
S_{P}=\left\{u \in U:\left|f_{j}(u)\right|=1, j=1, \cdots, n\right\} .
$$

Thus, it "lies over" the $n$-torus.

A number of people have recognized the value of an affirmative answer to the following

(III.0) Questron. If $L$ is a polynomially convex set, is it always possible to find $L_{i}$, a sequence of analytic polyhedra, converging ${ }^{9}$ to $L$, in such a way that the Silov boundaries, $S_{L_{i}}$, converge to $S_{L}$ ? Unfortunately, the answer is "no." This will be a consequence of the next few results.

(III.1) ThEOREM. Let $L$ be an analytic polyhedron. If $h$ is analytic about $L$ and $\log (h)$ is defined on $S_{L}$, then $h$ does not vanish at any point of $L$.

Moreover, this persists in the limit. That is,

(III.2) Corollary. Let $L_{i}$ be a sequence of analytic polyhedra converging to a compact set, $L$. Let $S$ be the limit set of the sequence, $S_{L_{i}}$. If $h$ is analytic about $L$ and $\log (h)$ is defined on $S$, then $h$ does not vanish at any point of $L$.

\footnotetext{
? Convergence in the usual Hausdorff topology for the compact subsets of $C^{n}$.
} 
(III.3) REMARK. It follows, that if $S$ is simply-coconnected and rationally convex, then $S=L$. Compare this with Question II.7.

However, there is an

(III.4) EXAMPLE. There is a polynomially convex set, $L$, in $C^{2}$, such that the coordinate function, $z_{1}$, vanishes at a point of $L$, even though $\log \left(z_{1}\right)$ is defined on $S_{L}$.

Such an example (in some $C^{n}$ ) was first constructed by K. Hoffman to refute a conjecture ${ }^{10}$ of the author. Later, E. Bishop suggested the following, extremely simple, example. Let $E=E_{1} \cup E_{2}$, where $E_{1}=\left\{\left(e^{i \theta}, z_{2}\right): 0 \leqq \theta \leqq \pi,\left|z_{2}\right|=1\right\}$ and $E_{2}=\left\{\left(e^{i \theta}, 0\right): \pi \leqq \theta \leqq 2 \pi\right\}$. If we set $L=\operatorname{hull}(E)$, then $S_{L}$ is contained in $E$. Since $E_{1}$ and $E_{2}$ are disjoint, and $\log \left(z_{1}\right)$ is defined on each, it follows that $\log \left(z_{1}\right)$ is defined on $S_{L}$. But it is easily verified that $\operatorname{hull}(E)$ contains $(0,0)$, at which point $z_{1}$ vanishes.

Clearly, III.2 and III.4 together give a "no" answer to Question III.0.

We can also use the $E$ of Example III.4 to show

(III.5) There is a rational polyhedron (a compact set defined by a finite number of rational inequalities) whose polynomial convex hull is not an analytic polyhedron.

For, $E$ is rationally convex and is, therefore, a decreasing intersection of rational polyhedra. Hence, there is a rational polyhedron, $R$, which contains $E$ and on which $\log \left(z_{1}\right)$ is defined. Since hull $(R)$ must contain $(0,0)$, we may now apply Theorem III.1 to deduce that $\operatorname{hull}(R)$ is not an analytic polyhedron.

IV. A hull with no analytic structure. The set hull $(X)$ is defined by a certain maximum modulus relation (see $\S 0$ ). Is this anything more than the classical maximum modulus principle for analytic functions on an analytic variety? In particular, does the set hull $(X)-X$ consist of (or, at least, contain) positive dimensional analytic varieties? For some fairly general cases, the analytic varieties making up hull $(X)-X$ have been exhibited. (See $[3 ; 4 ; 10 ; 12]$.) However, our result is

(IV.1) Theorem. ${ }^{11}$ There is a compact set, $X$, in $C^{2}$, such that $X \neq$ hull $(X)$, but hull $(X)$ does not contain any positive dimensional analytic varieties.

Our approach is to construct an $X \neq \operatorname{hull}(X)$, such that neither one of the coordinate projections, $z_{1}($ hull $(X)), z_{2}$ (hull $\left.(X)\right)$, contains any open subset of the plane. It follows from the open mapping property

${ }^{10}$ Namely, that there was no such example!

${ }^{11} \mathrm{~A}$ proof is given in [8]. 
of analytic functions on an analytic variety, that hull $(X)$ cannot contain any analytic varieties (of positive dimension).

Acknowledgments. We are indebted to E. Bishop and K. Hoffman for their examples used in answering Question III.0. Also, we benefited greatly from the advice of both K. Hoffman and I. M. Singer while working on parts of $\S \S I$ and IV. Finally, we were guided, at many points, by works of J. Wermer $[9 ; 10 ; 11]$.

All proofs will appear elsewhere.

\section{REFERENCES}

1. E. Bishop, A minimal boundary for function algebras, Pacific J. Math. 9 (1959), 629-642. 242.

2. - Mappings of partially analytic spaces, Amer. J. Math. 83 (1961), 209-

3. - Analyticity in certain Banach algebras, Trans. Amer. Math. Soc. 102 (1962), 507-544.

4. K. Hoffman, Minimal boundaries for analytic polyhedra, Rend. Circ. Mat. Palermo 9 (1960), 147-160.

5. K. Oka, Sur les fonctions analytiques de plusieurs variables. I. Domaines convexes par rapport aux fonctions rationelles, J. Sci. Hiroshima Univ. Ser. A 6 (1936), 245-255.

6. - Sur les fonctions analytiques de plusieurs variables. II. Domaines d'holomorphie, J. Sci. Hiroshima Univ. Ser. A 7 (1937), 115-130.

7. H. Rossi, The local maximum modulus principle, Ann. of Math. 72 (1960), 1-11.

8. G. Stolzenberg, $A$ hull with no analytic structure, J. Math. Mech. (to appear).

9. J. Wermer, Polynomial approximation on an arc in $C^{3}$, Ann. of Math. 62 (1955), 269-270.

10. - The hull of a curve in $C^{n}$, Ann. of Math. 68 (1958), 550-561.

11. An example concerning polynomial convexity, Math. Ann. 139 (1959), $147-150$.

12. - Dirichlet algebras, Duke Math. J. 27 (1960), 373-382. 1961.

13. - Banach algebras and analytic functions, Academic Press, New York,

HARVARD UNIVERSITY 